\title{
The Nonlinear Theory of Elastic Shells with Phase Transitions
}

\author{
VICTOR A. EREMEYEV ${ }^{1}$ and WOJCIECH PIETRASZKIEWICZ ${ }^{2}$ \\ ${ }^{1}$ Rostov State University, Zorge str. 5, 344090 Rostov on Don, Russia. \\ E-mail: eremeyev@math.rsu.ru \\ ${ }^{2}$ Institute of Fluid-Flow Machinery of the Polish Academy of Sciences, ul. Fiszera 14, \\ 80-952 Gdanss, Poland. E-mail: pietrasz@imp.gda.pl
}

\begin{abstract}
We develop the general nonlinear theory of elastic shells with an account of phase transitions in the shell material. Our formulation is based on the dynamically and kinematically exact through-the-thickness reduction of three-dimensional description of the phenomenon to the twodimensional form written on the shell base surface. In this model shell displacements are expressed by work-averaged translations and rotations of the shell cross-sections. All shell relations are then found from the variational principle of the stationary total potential energy. In particular, we derive the new global dynamic continuity condition at the singular surface curve modelling the phase interface. We also discuss particular forms of the local dynamic continuity conditions at coherent and incoherent interface curves. The results are illustrated by an example of a phase transition in an infinite plate with a circular hole.
\end{abstract}

\section{Introduction}

Since the pioneering paper by Gibbs [1], phase transformation phenomena in threedimensional (3D) continua have been described by introducing into the body a movable singular surface separating two different material phases in thermodynamic equilibrium state. The main results are summarised in several books, for example [2-6], where many references to original papers are given.

The phase transformation phenomenon manifests itself best in thin layers of matter: films, membranes, plates, and shells. For example, thin films made of shape-memory alloys like $\mathrm{NiTi}, \mathrm{NiMnGa}, \mathrm{NiTiCu}$, or $\mathrm{NiAl}$ can considerably alter their shapes under appropriate stress and/or temperature changes. Full analysis of the phenomenon in such thin-walled structures is often infeasible if one wants to apply the 3D continuum model. The mechanical description of behaviour of such structures can conveniently be based on various $2 \mathrm{D}$ shell models consisting of a base surface endowed with various fields modelling an additional microstructure. Then the notion of a movable surface curve separating shell regions with differ- 
ent material phases is an appropriate and convenient tool for modelling the phase interface in thin-walled shell structures. It is surprising, however, that we are not aware of any complete theoretical model of phase transitions in shells proposed in the literature. A Cosserat-type membrane with one director was proposed in $[7,8]$ to model single crystal thin films of martensitic materials. However, neither the rotation about the director was included nor dynamic continuity conditions at the curvilinear phase interface were discused in $[7,8]$.

The aim of this paper is to formulate the general nonlinear theory of shells with an account of occurence of phase transformation in the shell material. One might approach this problem by analysing first some test examples with various simplified shell models. Among such shell models let us mention as examples the linear, the geometrically nonlinear, or the fully nonlinear theories of shells based on the membrane model, the Kirchhoff-Love type model, or the Timoshenko-Reissner type model. However, we want here to forego all possible simplified 2D models of thin-walled shell structures and apply at once the most general approach based on the dynamically and kinematically exact shell model. The model is based on an exact through-the-thickness reduction of the 3D formulation of the problem to the $2 \mathrm{D}$ one, and is expressed through stress resultants and work-averaged deformation fields defined on the shell base surface.

In Section 2 we recall basic nonlinear relations of regular shell structures. The local equilibrium equations and dynamic boundary conditions (5) are derived in the most general form in terms of stress and couple resultants using an exact throughthe-thickness integration of 3D balance laws of the linear and angular momentum of continuum mechanics [9-15]. The corresponding shell kinematics is then established as an energetically exact dual structure from the virtual work identity (8). As a result, the shell displacements are expressed by a work-averaged translation vector and a work-averaged rotation tensor (9) as two independent field variables describing the gross deformation of the shell cross-sections. The shell strain and bending measures (11) and their virtual changes (12) are energetically exact consequences of the exact local equilibrium equations (5) $)_{1}$. Approximations of the shell model are included only in the constitutive equations of the elastic material (15), which are approximate experimental physical laws anyway. Such a general shell model having the structure of the classical Cosserat surface [16-21] comprises all other simplified shell models as special cases. For this general nonlinear shell model efficient finite element algorithms and computer programs were developed and several test examples of equilibrium, stability and dynamics of complex shell structures were solved [15, 22-25].

The nonlinear equilibrium problem of elastic shells with phase transitions is formulated in Section 3 as the stationary problem for the functional (22) of total potential energy. In the functional the position of the singular surface curve modelling the phase interface is not known in advance. Stationary conditions of the functional lead to the local equilibrium conditions given in Section 2, but additionally give us the global continuity condition (27) at the interface. 
We discuss in more detail coherent and incoherent curvilinear phase interfaces. At the coherent interface both translations and rotations are assumed to be continuous, while at the incoherent interface the continuity of rotations can be violated. For both cases we derive special forms (37) and (42) of the local dynamic continuity conditions. In particular, one of each of the conditions (37) and (42) represents an additional relation allowing one to establish the position of the interface in the thermomechanic equilibrium state of phase transition in shells.

The results obtained here are illustrated by an example of phase transition in an infinite plate with a circular hole.

\section{Notation and Exact Relations for Regular Shells}

The shell is the three-dimensional (3D) solid body which in a reference (undeformed) configuration is identified with a region $B$ of the physical space $\&$ with the translation vector space $E$. It is assumed that geometry of $B$ can be uniquely described in the normal coordinates $\left(\theta^{\alpha}, \zeta\right), \alpha=1,2$, where $\zeta=0$ defines the regular shell base surface $M \subset B$, and $-h^{-} \leqslant \zeta \leqslant h^{+}$is the distance from $M$, with $h=h^{-}+h^{+}$the shell thickness. Relative to an inertial frame $\left(\mathrm{o}, \mathbf{i}_{k}\right)$, where o $\in \mathcal{E}$, and $\mathbf{i}_{k} \in E, k=1,2,3$, are orthonormal 3D vectors, the position vector $\mathbf{p}$ of an arbitrary point $\mathrm{p} \in B$ is given by

$$
\mathbf{p}\left(\theta^{\alpha}, \zeta\right)=\boldsymbol{x}\left(\theta^{\alpha}\right)+\zeta \boldsymbol{n}\left(\theta^{\alpha}\right),
$$

where $\boldsymbol{x}\left(\theta^{\alpha}\right)=\mathbf{p}\left(\theta^{\alpha}, 0\right)$ is the position vector of a point $x \in M$, and $\boldsymbol{n}\left(\theta^{\alpha}\right)$ is the unit normal vector orienting $M$. The surface covariant and contravariant base vectors $\boldsymbol{a}_{\alpha}$ and $\boldsymbol{a}^{\beta}$ are defined by

$$
\boldsymbol{a}_{\alpha}=\frac{\partial \boldsymbol{x}}{\partial \theta^{\alpha}} \equiv \boldsymbol{x}_{, \alpha}, \quad \boldsymbol{a}^{\beta} \cdot \boldsymbol{a}_{\alpha}=\delta_{\alpha}^{\beta}, \quad \boldsymbol{n}=\frac{1}{2} \epsilon^{\alpha \beta} \boldsymbol{a}_{\alpha} \times \boldsymbol{a}_{\beta},
$$

where $\epsilon^{\alpha \beta}$ are components of the surface permutation tensor $\epsilon \in T_{x} M \otimes T_{x} M, \otimes$ is the tensor product, and $T_{x} M$ is the tangent space to $M$ at $x \in M$.

During shell deformation $\chi$, assumed to be continuous and one-to-one, the undeformed base surface $M$ is transformed into the deformed one $N=\chi(M)$ with the position vector $\boldsymbol{y}=\chi(\boldsymbol{x})$ parametrised again by the surface convected coordinates, $\boldsymbol{y}=\boldsymbol{y}\left(\theta^{\alpha}\right)$.

Equilibrium equations and dynamic boundary conditions of the general nonlinear theory of shells can be derived exactly [9-15] by direct through-the-thickness integration of 3D balance laws of linear and angular momentum of continuum mechanics [26]. In solid mechanics it is usual to apply the Lagrangian description relative to the undeformed base surface $M$. In the equilibrium shell problem discussed here the global equilibrium conditions require the total force and total torque of all loads acting upon any part $\Pi \subset M$ to vanish (see [14], (2.200))

$$
\iint_{\Pi} \boldsymbol{f} \mathrm{da}+\int_{\partial \Pi \backslash \partial M_{f}} \boldsymbol{n}_{v} \mathrm{~d} s+\int_{\partial \Pi \cap \partial M_{f}} \boldsymbol{n}^{*} \mathrm{~d} s=\mathbf{0},
$$




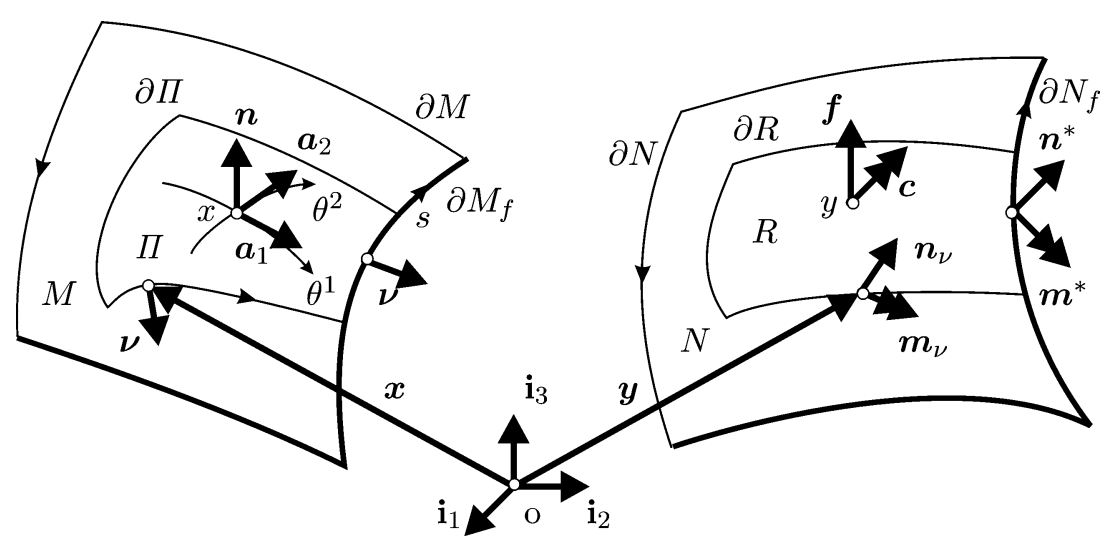

Figure 1. Shell kinematics.

$$
\begin{aligned}
& \iint_{\Pi}(\boldsymbol{c}+\boldsymbol{y} \times \boldsymbol{f}) \mathrm{d} a+\int_{\partial \Pi \backslash \partial M_{f}}\left(\boldsymbol{m}_{v}+\boldsymbol{y} \times \boldsymbol{n}_{v}\right) \mathrm{d} s \\
& \quad+\int_{\partial \Pi \cap \partial M_{f}}\left(\boldsymbol{m}^{*}+\boldsymbol{y} \times \boldsymbol{n}^{*}\right) \mathrm{d} s=\mathbf{0} .
\end{aligned}
$$

In (3), see Figure $1, \boldsymbol{f}$ and $\boldsymbol{c}$ are the external surface resultant force and couple vectors applied at any point $\boldsymbol{y} \in N$, but measured per unit area of $M$. Similarly, $\boldsymbol{n}_{v}$ and $\boldsymbol{m}_{v}$ are the internal contact stress and couple resultant vectors defined at an arbitrary boundary $\partial R$ of $R=\chi(\Pi)$, while $n^{*}$ and $\boldsymbol{m}^{*}$ are the external boundary resultant force and couple vectors applied along the part $\partial N_{f}$ of $N=\chi(M)$, respectively. The latter four vectors are measured per unit length of the undeformed boundaries $\partial \Pi$ or $\partial M_{f}$, respectively, with the unit external normal vector $\boldsymbol{v} \in$ $T_{x} M$.

According to the Cauchy postulate, the contact vectors $\boldsymbol{n}_{v}$ and $\boldsymbol{m}_{v}$ can be represented through the respective internal surface stress and couple resultant tensors $\boldsymbol{N}$ and $\boldsymbol{M}$ by

$$
\boldsymbol{n}_{v}=\boldsymbol{N} \boldsymbol{v}=\boldsymbol{n}^{\alpha} v_{\alpha}, \quad \boldsymbol{m}_{v}=\boldsymbol{M} \boldsymbol{v}=\boldsymbol{m}^{\alpha} v_{\alpha},
$$

where $v_{\alpha}=\boldsymbol{v} \cdot \boldsymbol{a}_{\alpha}$, while $\boldsymbol{N}=\boldsymbol{n}^{\alpha} \otimes \boldsymbol{a}_{\alpha}$ and $\boldsymbol{M}=\boldsymbol{m}^{\alpha} \otimes \boldsymbol{a}_{\alpha}$. The tensors $\boldsymbol{N} \in$ $E \otimes T_{x} M$ and $M \in E \otimes T_{x} M$ defined on $M$ are some analogues in shell theory of the first Piola-Kirchhoff stress tensor $\mathbf{T}_{R} \in E \otimes E$ defined on $B$ in $3 \mathrm{D}$ continuum mechanics [26].

Applying the Stokes theorem to the second terms of (3) and using the representations (4) we obtain the usual local equilibrium equations and dynamic boundary conditions $[9,10]$

$$
\begin{array}{lll}
\left.\boldsymbol{n}^{\alpha}\right|_{\alpha}+\boldsymbol{f}=\mathbf{0}, & \left.\boldsymbol{m}^{\alpha}\right|_{\alpha}+\boldsymbol{y}_{, \alpha} \times \boldsymbol{n}^{\alpha}+\boldsymbol{c}=\mathbf{0} & \text { in } M, \\
\boldsymbol{n}^{\alpha} v_{\alpha}-\boldsymbol{n}^{*}=\mathbf{0}, & \boldsymbol{m}^{\alpha} v_{\alpha}-\boldsymbol{m}^{*}=\mathbf{0} & \text { along } \partial M_{f},
\end{array}
$$

where (.) $\left.\right|_{\alpha}$ is the covariant derivative in the undeformed surface metric $A_{\alpha \beta}=$ $\boldsymbol{a}_{\alpha} \cdot \boldsymbol{a}_{\beta}$. 
In the coordinate-free notation the local equilibrium conditions (5) take the form $[15,27]$

$$
\begin{array}{lll}
D i v_{s} \boldsymbol{N}+\boldsymbol{f}=\mathbf{0}, & D i v_{s} \boldsymbol{M}+\operatorname{ax}\left(\boldsymbol{N} \boldsymbol{F}^{\mathrm{T}}-\boldsymbol{F} \boldsymbol{N}^{\mathrm{T}}\right)+\boldsymbol{c}=\mathbf{0} & \text { in } M, \\
\boldsymbol{N} \boldsymbol{v}-\boldsymbol{n}^{*}=\mathbf{0}, & \boldsymbol{M} \boldsymbol{v}-\boldsymbol{m}^{*}=\mathbf{0} & \text { along } \partial M_{f},
\end{array}
$$

where $\boldsymbol{F}=\operatorname{Grad}_{s} \boldsymbol{y}=\boldsymbol{y}_{, \alpha} \otimes \boldsymbol{a}^{\alpha} \in E \otimes T_{x} M$ is the surface gradient of deformation $\chi(\boldsymbol{x}), \operatorname{ax}\left(\boldsymbol{N} \boldsymbol{F}^{\mathrm{T}}-\boldsymbol{F} \boldsymbol{N}^{\mathrm{T}}\right)$ denotes the axial vector associated with the skew tensor $\boldsymbol{N} \boldsymbol{F}^{\mathrm{T}}-\boldsymbol{F} \boldsymbol{N}^{\mathrm{T}} \in E \otimes E$, while $\mathrm{Grad}_{s}$ and $\operatorname{Div}_{s}$ are the surface gradient and divergence operators on $M$, respectively, defined intrinsically in [28-30].

The equilibrium conditions (5) or (6) are exact consequences of 3D balance of forces acting on any part of the shell-like body $B$.

Let $\boldsymbol{v}$ and $\boldsymbol{w}$ be two arbitrary smooth vector fields on $M$. We can set the integral identity

$$
\begin{gathered}
\iint_{M}\left\{\left(\left.\boldsymbol{n}^{\alpha}\right|_{\alpha}+\boldsymbol{f}\right) \cdot \boldsymbol{v}+\left(\left.\boldsymbol{m}^{\alpha}\right|_{\alpha}+\boldsymbol{y}_{, \alpha} \times \boldsymbol{n}^{\alpha}+\boldsymbol{c}\right) \cdot \boldsymbol{w}\right\} \mathrm{d} a \\
-\int_{\partial M_{f}}\left\{\left(\boldsymbol{n}^{\alpha} v_{\alpha}-\boldsymbol{n}^{*}\right) \cdot \boldsymbol{v}+\left(\boldsymbol{m}^{\alpha} v_{\alpha}-\boldsymbol{m}^{*}\right) \cdot \boldsymbol{w}\right\} \mathrm{d} s=0 .
\end{gathered}
$$

The identity can be transformed with the help of the Stokes theorem to the form

$$
\begin{aligned}
& \iint_{M}\left\{\boldsymbol{n}^{\alpha} \cdot\left(\boldsymbol{v}_{, \alpha}+\boldsymbol{y}_{, \alpha} \times \boldsymbol{w}\right)+\boldsymbol{m}^{\alpha} \cdot \boldsymbol{w}_{, \alpha}\right\} \mathrm{d} a \\
& =\iint_{M}(\boldsymbol{f} \cdot \boldsymbol{v}+\boldsymbol{c} \cdot \boldsymbol{w}) \mathrm{d} a+\int_{\partial M_{f}}\left(\boldsymbol{n}^{*} \cdot \boldsymbol{v}+\boldsymbol{m}^{*} \cdot \boldsymbol{w}\right) \mathrm{d} s \\
& \quad+\int_{\partial M_{d}}\left(\boldsymbol{n}^{\alpha} v_{\alpha} \cdot \boldsymbol{v}+\boldsymbol{m}^{\alpha} v_{\alpha} \cdot \boldsymbol{w}\right) \mathrm{d} s,
\end{aligned}
$$

where $\partial M_{d}=\partial M \backslash \partial M_{f}$.

If $\boldsymbol{v}$ and $\boldsymbol{w}$ are interpreted as kinematically admissible virtual translation and rotation vectors such that $\boldsymbol{v}=\boldsymbol{w}=\mathbf{0}$ along $\partial M_{d}$, then the last line integral identically vanishes. Two integrals in the second row of (8) can be interpreted as the external virtual work performed by the given surface $f, c$ and boundary $\boldsymbol{n}^{*}, \boldsymbol{m}^{*}$ loads, respectively. In this context the first surface integral of (8) has the meaning of the internal virtual work, where the expressions $\boldsymbol{v}_{, \alpha}+\boldsymbol{y}_{, \alpha} \times \boldsymbol{w}$ and $\boldsymbol{w}_{, \alpha}$ are just virtual changes of appropriately defined shell strain and bending vectors, respectively. Then the formula (8) takes the meaning of the principle of virtual work for the shell.

Let the vector field $\boldsymbol{u}(\boldsymbol{x})$ represent the work-averaged translations and the proper orthogonal tensor field $\boldsymbol{Q}(\boldsymbol{x})$ the work-averaged rotations of the shell cross sections $[9,10,12]$. The deformed shell configuration can then be described by the relations

$$
y=\chi(x)=x+u, \quad d_{i}=\varphi(x)=Q a_{i},
$$


where $\boldsymbol{a}_{3} \equiv \boldsymbol{n}$, and $\boldsymbol{d}_{i}=\left(\boldsymbol{d}_{\alpha}, \boldsymbol{d}\right)$ are three directors attached to any point of the deformed base surface $N=\chi(M)$.

Let us consider a one-parametric family of shell deformations described by $\chi(\boldsymbol{x}, t)=\boldsymbol{x}+\boldsymbol{u}(\boldsymbol{x}, t)$ and $\boldsymbol{Q}(\boldsymbol{x}, t)=\boldsymbol{d}_{i}(\boldsymbol{x}, t) \otimes \boldsymbol{a}^{i}(\boldsymbol{x})$, where $t$ is a scalar (time-like) parameter such that $t=0$ corresponds to the undeformed shell configuration and $t$ to the deformed one. Then the vectors $\boldsymbol{v}$ and $\boldsymbol{w}$ in (7) can be interpreted as virtual changes of $\boldsymbol{u}$ and $\boldsymbol{Q}$ (linear and angular velocities in a real motion) according to

$$
\begin{aligned}
& \boldsymbol{v}=\frac{\partial \chi(\boldsymbol{x}, t)}{\partial t}=\dot{\boldsymbol{u}}(\boldsymbol{x}, t) \equiv \delta \boldsymbol{u}=v_{\alpha} \boldsymbol{d}^{\alpha}+v \boldsymbol{d}, \\
& \boldsymbol{w}=\operatorname{ax}\left(\dot{\boldsymbol{Q}}(\boldsymbol{x}, t) \boldsymbol{Q}^{\mathrm{T}}(\boldsymbol{x}, t)\right) \equiv \operatorname{ax}\left(\delta \boldsymbol{Q} \boldsymbol{Q}^{\mathrm{T}}\right)=w_{\alpha} \boldsymbol{d}^{\alpha}+w \boldsymbol{d},
\end{aligned}
$$

where $\delta$ is the symbol of variation.

The shell strain $\boldsymbol{\varepsilon}_{\alpha}$ and bending $\boldsymbol{\varkappa}_{\alpha}$ vectors corresponding to the kinematics (9) and their virtual changes can be defined by $[12,14]$

$$
\begin{aligned}
& \boldsymbol{\varepsilon}_{\alpha}=\boldsymbol{y}_{, \alpha}-\boldsymbol{d}_{\alpha}=\boldsymbol{u},_{\alpha}+(\mathbf{1}-\boldsymbol{Q}) \boldsymbol{a}_{\alpha}=E_{\alpha \beta} \boldsymbol{d}^{\beta}+E_{\alpha} \boldsymbol{d}, \\
& \boldsymbol{x}_{\alpha}=\operatorname{ax}\left(\boldsymbol{Q}_{, \alpha} \boldsymbol{Q}^{\mathrm{T}}\right)=\frac{1}{2} \boldsymbol{d}^{i} \times \boldsymbol{Q}_{, \alpha} \boldsymbol{Q}^{\mathrm{T}} \boldsymbol{d}_{i}=\boldsymbol{d} \times K_{\alpha \beta} \boldsymbol{d}^{\beta}+K_{\alpha} \boldsymbol{d}, \\
& \delta^{c} \boldsymbol{\varepsilon}_{\alpha}=\boldsymbol{v}_{, \alpha}+\boldsymbol{y}_{, \alpha} \times \boldsymbol{w}=\delta E_{\alpha \beta} \boldsymbol{d}^{\beta}+\delta E_{\alpha} \boldsymbol{d}, \\
& \delta^{c} \boldsymbol{\varkappa}_{\alpha}=\boldsymbol{w}_{, \alpha}=\boldsymbol{d} \times \delta K_{\alpha \beta} \boldsymbol{d}^{\beta}+\delta K_{\alpha} \boldsymbol{d},
\end{aligned}
$$

where $\delta^{c}(\cdot)=\boldsymbol{Q}\left\{\delta\left(\boldsymbol{Q}^{\mathrm{T}}(\cdot)\right)\right\}$ is the co-rotational variation (the co-rotational time derivative in a real motion), and $\mathbf{1} \in E \otimes E$ is the metric tensor of the $3 \mathrm{D}$ vector space.

It follows from (12) and (8) that the surface vector strain measures $\boldsymbol{\varepsilon}_{\alpha}, \boldsymbol{\varkappa}_{\alpha}$ are work-conjugate to the respective exact shell vector stress measures $\boldsymbol{n}^{\alpha}, \boldsymbol{m}^{\alpha}$. As a result, the shell kinematics consisting of the displacements $\boldsymbol{u}, \boldsymbol{Q}$ satisfying (9) and of the strain measures $\boldsymbol{\varepsilon}_{\alpha}, \boldsymbol{\varkappa}_{\alpha}$ satisfying (12) is again an energetically exact consequence of the exact local equilibrium equations $(5)_{1}$. The shell kinematics is valid for unrestricted translations, rotations, strains, and/or bendings of the shell material elements, and does not depend on the shell thickness, internal throughthe-thickness structure, and/or material properties.

The kinematic structure (9)-(12) of the general shell theory is identical with that of the classical version of the Cosserat surface [16-21]. The kinematic structure (9)-(12) differs from kinematics of the surface with one or more attached deformable directors, often also called the Cosserat surface, see, for example, [31-33]. Moreover, the kinematic structure following from (9)-(12) is also different from kinematics of the nonlinear theory of shells based on the Kirchhoff-Love [14, 30, 34-37] or Timoshenko-Reissner-type [32, 38, 39] constraints.

For a shell made of an elastic material there should exist a strain energy density $W\left(\boldsymbol{\varepsilon}_{\alpha}, \boldsymbol{x}_{\alpha}\right)$ such that

$$
\boldsymbol{n}^{\alpha} \cdot \delta^{c} \boldsymbol{\varepsilon}_{\alpha}+\boldsymbol{m}^{\alpha} \cdot \delta^{c} \boldsymbol{\varkappa}_{\alpha}=\delta W .
$$


Representing $\boldsymbol{n}^{\alpha}$ and $\boldsymbol{m}^{\alpha}$ naturally though their components along $\boldsymbol{d}_{i}$ we obtain

$$
\boldsymbol{n}^{\alpha}=N^{\alpha \beta} \boldsymbol{d}_{\beta}+N^{\alpha} \boldsymbol{d}, \quad \boldsymbol{m}^{\alpha}=\boldsymbol{d} \times M^{\alpha \beta} \boldsymbol{d}_{\beta}+M^{\alpha} \boldsymbol{d} .
$$

From the principle of material frame-indifference we can find that, in fact, $W=$ $W\left(E_{\alpha \beta}, E_{\alpha}, K_{\alpha \beta}, K_{\alpha}\right)$, from which we obtain the following constitutive equations:

$$
N^{\alpha \beta}=\frac{\partial W}{\partial E_{\alpha \beta}}, \quad N^{\alpha}=\frac{\partial W}{\partial E_{\alpha}}, \quad M^{\alpha \beta}=\frac{\partial W}{\partial K_{\alpha \beta}}, \quad M^{\alpha}=\frac{\partial W}{\partial K_{\alpha}} .
$$

Some possible quadratic forms of the strain energy density $W$ were briefly discussed by Libai and Simmonds [10]. In the case of an unsymmetric throughthe-thickness laminate the generally anisotropic and algebraically homogeneous expression for $W$ contains many terms and requires more than one hundred material constants to be known. If $W$ is assumed not to depend on curvature of $M$, for a symmetric laminate we can apply the theory of invariants [40] and show [21] that $W$ can be simplified to the form

$$
\begin{aligned}
W= & C_{1} E_{\alpha \beta} E^{\alpha \beta}+C_{2} E_{\alpha \beta} E^{\beta \alpha}+C_{3} E_{\alpha}^{\alpha} E_{\beta}^{\beta}+C_{4} E_{\alpha} E^{\alpha} \\
& +D_{1} K_{\alpha \beta} K^{\alpha \beta}+D_{2} K_{\alpha \beta} K^{\beta \alpha}+D_{3} K_{\alpha}^{\alpha} K_{\beta}^{\beta}+D_{4} K_{\alpha} K^{\alpha},
\end{aligned}
$$

where $C_{n}, D_{n}, n=1,2,3,4$, are material constants to be found by experiments.

When strains in the shell space are small, then we can apply the linear constitutive equations proposed in [22,23] which are extensions of the classical constitutive equations based on the consistent first approximation theory:

$$
\begin{array}{ll}
N^{\alpha \beta}=C\left[(1-v) E^{\alpha \beta}+v A^{\alpha \beta} E_{\varkappa}^{\varkappa}\right], & N^{\alpha}=\frac{1}{2} \alpha_{s} C(1-v) E^{\alpha}, \\
M^{\alpha \beta}=D\left[(1-v) K^{\alpha \beta}+v A^{\alpha \beta} K_{\varkappa}^{\varkappa}\right], & M^{\alpha}=\alpha_{t} D(1-v) K^{\alpha}, \\
C=\frac{E h}{1-v^{2}}, \quad D=\frac{E h^{3}}{12\left(1-v^{2}\right)}, & N^{\alpha \beta} \neq N^{\beta \alpha}, \quad K^{\alpha \beta} \neq K^{\beta \alpha}, \quad \text { etc. }
\end{array}
$$

Here $E$ is the Young modulus and $v$ the Poisson ratio of the material, while $\alpha_{s}$ and $\alpha_{t}$ are shear and torsional coefficients, respectively. It is seen that (17) would follow from differentiation of (16) if $C_{1}=C(1-v), C_{2}=0, C_{3}=C v, C_{4}=\frac{1}{2} \alpha_{s} C(1-v)$, $D_{1}=D(1-v), D_{2}=0, D_{3}=D v, D_{4}=\alpha_{t} D(1-v)$.

More accurate constitutive equations than (17) for shells undergoing small elastic strains can be found reformulating and extending the consistent second approximation to the strain energy density $[38,41]$.

Let the external surface and boundary loads have a potential $A: S_{a} \rightarrow \mathbb{R}$ such that

$$
\delta A=\iint_{M}(\boldsymbol{f} \cdot \boldsymbol{v}+\boldsymbol{c} \cdot \boldsymbol{w}) \mathrm{d} a+\int_{\partial M_{f}}\left(\boldsymbol{n}^{*} \cdot \boldsymbol{v}+\boldsymbol{m}^{*} \cdot \boldsymbol{w}\right) \mathrm{d} s,
$$

where $S_{a}$ is the configuration space of admissible fields $\boldsymbol{u}, \boldsymbol{Q}$ satisfying

$$
\boldsymbol{u}-\boldsymbol{u}^{*}=\mathbf{0}, \quad \boldsymbol{Q}-\boldsymbol{Q}^{*}=\mathbf{0} \quad \text { along } \partial M_{d} .
$$


The functions $\boldsymbol{u}^{*}(s)$ and $\boldsymbol{Q}^{*}(s)$ in (19) should be found from kinematic boundary conditions $\mathbf{u}(\mathrm{p})-\mathbf{u}^{*}(\mathrm{p})=\mathbf{0}$ of the $3 \mathrm{D}$ shell-like body prescribed at any point $\mathrm{p}(s, \zeta)$ of the lateral shell boundary surface $\partial \boldsymbol{B}_{d}$. The functions $\boldsymbol{u}^{*}(s)$ and $\boldsymbol{Q}^{*}(s)$ should be such that the work done along $\partial M_{d}$ by the resultant stress and couple vectors $\boldsymbol{n}_{v}, \boldsymbol{m}_{v}$ on the respective $\boldsymbol{u}^{*}, \boldsymbol{Q}^{*}$ be the same as the work done at $\partial B_{d}$ by the nominal 3D stress vector $\mathbf{t}_{n}(\mathrm{p})$ on the translation $\mathbf{u}^{*}(\mathrm{p})$ prescribed at $\mathrm{p}(s, \zeta) \in \partial B_{d}$.

In various shell problems a mixed form of boundary conditions can be applied along $\partial M$ by prescribing six pairwise work-conjugate load or displacement components in the basis $\boldsymbol{v}, \boldsymbol{\tau}, \boldsymbol{n}$. In such a case division of $\partial M$ into disjoint parts $\partial M_{f}$ and $\partial M_{d}$ is understood to be made separately for each of the six components of boundary conditions.

Expressing (11) through $\boldsymbol{u}, \boldsymbol{Q}$ by (9) we can introduce the functional

$$
I(\boldsymbol{u}, \boldsymbol{Q})=\iint_{M} W(\boldsymbol{u}, \boldsymbol{Q}) \mathrm{d} a-A(\boldsymbol{u}, \boldsymbol{Q})
$$

such that the variational principle of the stationary total potential energy takes the form

$$
\delta I(\boldsymbol{u}, \boldsymbol{Q})=0, \quad \forall(\boldsymbol{u}, \boldsymbol{Q}) \in S_{a} .
$$

The principle states that among all $(\boldsymbol{u}, \boldsymbol{Q}) \in S_{a}$ only those render the functional $I(\boldsymbol{u}, \boldsymbol{Q})$ stationary which satisfy the local equilibrium conditions (5).

\section{Singular Curve in the Two-Phase Elastic Shell}

Some fields on $M$ defined in Section 2 or their surface gradients may suffer jump discontinuities at a surface curve $C \subset M$ called a singular curve. The curve $C$ can be defined parametrically by the position vector $\boldsymbol{x}_{c}(s)=\boldsymbol{x}_{c}\left\{\theta^{\alpha}(s)\right\}$, where $s$ is the arc length along $C$. The curve $C$ divides the surface $M$ into two parts, $M_{A}$ and $M_{B}$, such that $M=M_{A} \cup M_{B}$ and $C=M_{A} \cap M_{B}$, Figure 2 .

At each regular point $x_{c} \in C$ we can introduce the unit tangent vector $\tau=$ $\mathrm{d} \boldsymbol{x}_{c} / \mathrm{d} s \equiv \boldsymbol{x}_{c}^{\prime}$, the unit normal vector $\boldsymbol{n}$ following from orientation of $M$, and the external unit normal vector $\boldsymbol{v}=\boldsymbol{\tau} \times \boldsymbol{n}$. When the orientation of $C$ is assumed to coincide with that of $\partial M_{A}$, for which $\tau_{A}$ and $\boldsymbol{v}_{A}$ are respective unit tangent and outward normal vectors, then $\boldsymbol{\tau}=\boldsymbol{\tau}_{A}$, and $\boldsymbol{v}=\boldsymbol{v}_{A}$ points outward from $M_{A}$.

From a continuum physics point of view, an important example of a shell with a singular curve is a shell undergoing a stress-induced phase transition possible, for example, in thin films made of martensitic materials. Consider a two-phase elastic shell such that in the deformed equilibrium state the material phases are assumed to appear separately in two closed complementary subregions $N_{A}$ and $N_{B}$ with the curvilinear phase interface $D$, Figure 2. The position of $D$ in the deformed configuration is given by the position vector $\boldsymbol{y}_{c}(s)$. Within the Lagrangian description and for the continuous one-to-one deformation $\chi$ we can introduce the singular curve $C$ in $M$ with the position vector $\boldsymbol{x}_{c}(s)$ being an image of $\boldsymbol{y}_{c}(s)$ under $\chi^{-1}$ such that 


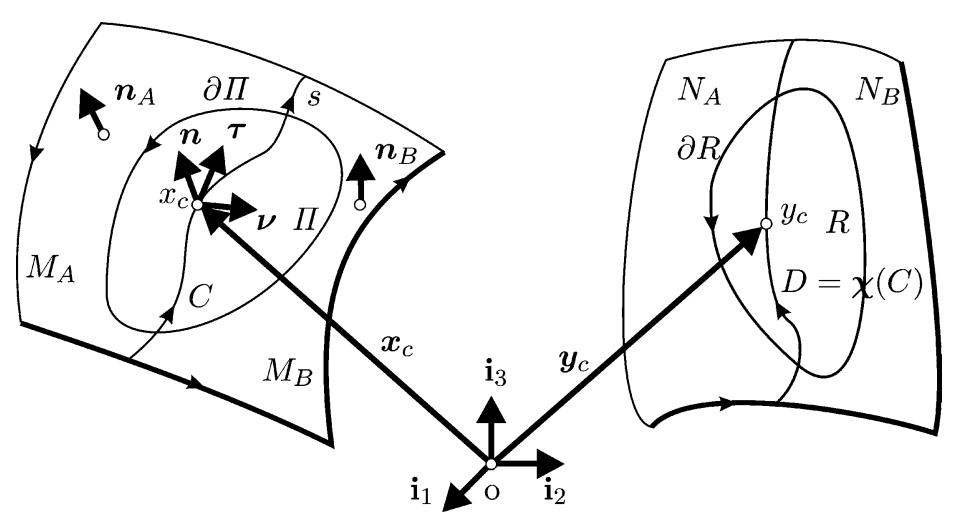

Figure 2. Two-phase shell kinematics.

$\boldsymbol{x}_{c}(s)=\chi^{-1}\left(\boldsymbol{y}_{c}(s)\right)$. Then the corresponding closed complementary subregions of $M$ are defined by $M_{A}=\chi^{-1}\left(N_{A}\right)$ and $M_{B}=\chi^{-1}\left(N_{B}\right)$.

In order to account for a possible phase transition of the material at the singular curve $C$, let us write the functional of the total potentional energy (20) in the form

$$
I=\iint_{M_{A}} W \mathrm{~d} a+\iint_{M_{B}} W \mathrm{~d} a-A,
$$

with possibly different expressions given in $M_{A}$ and $M_{B}$ for the shell strain energy density $W\left(\boldsymbol{\varepsilon}_{\alpha}, \boldsymbol{\varkappa}_{\alpha}\right)$ describing material properties of different elastic phases in the shell.

The form (22) of the functional used here is the simplest one. It does not take into account some physical effects associated with properties of the interface itself. In the 3D case these are, for example, the interface surface tension, or other effects described by scalar, vector, or tensor fields prescribed on the surface interface, see $[6,42,43]$. In the shell theory discussed here such effects can be modelled by adding to (22) an integral along $C$ of some scalar, vector, or tensor fields. We might also allow $I$ to depend upon the fields depending explicitly on time and temperature in order to discuss problems of phase transition in shells within the thermodynamic setting. Many such possible extensions of (22) should be discussed separately.

In problems of phase transition the position vector $\boldsymbol{x}_{c}$ of the curve $C$ within the surface $M$ is not known in advance, in general, and should be found in the process of solution of the boundary value problem.

In analogy to the problem of thermodynamic equilibrium of 3D elastic bodies undergoing a phase transition $[2,3]$, the position of the phase interface $C$ within $M$ can be established from the variational principle $\delta I=0$. The principle states that among all possible values of translations, rotations, and positions of the phase interface curve $C$ the actual solution renders the functional (22) stationary.

To deduce the variation $\delta I$ of (22) let us consider a one-parameter family of shell deformations $\boldsymbol{y}(\boldsymbol{x}, t), \boldsymbol{Q}(\boldsymbol{x}, t)$, and position vectors of surface curves $C(t)$ described within $M$ by $\boldsymbol{x}_{c}(t)=\boldsymbol{\psi}(s, t)$, where $t$ is again a scalar (time-like) 
parameter. The value $t=0$ corresponds to an initial position of $C$ (if it exists) before deformation, and the value $t$ describes the position of $C$ in $M$ corresponding to its position in $N(t)$ in the final equilibrium state of phase transition such that $\boldsymbol{x}_{c}(s, t)=\chi^{-1}\left(\boldsymbol{y}_{c}(s, t), t\right)$.

If the curve $C(t)$ transverses an arbitrary part $\Pi \subset M$ with the relative velocity $\boldsymbol{v}_{c}(s, t)=\dot{\boldsymbol{x}}_{c}(s, t) \in T_{x} M$ then by the transport theorem [3,27]

$$
\begin{aligned}
& \frac{\mathrm{d}}{\mathrm{d} t} \iint_{\Pi \backslash C(t)} W \mathrm{~d} a=\iint_{\Pi \backslash C(t)} \dot{W} \mathrm{~d} a+\int_{\Pi \cap C(t)}\left[\left(\boldsymbol{v}_{c} \cdot \boldsymbol{v}\right) W\right] \mathrm{d} s, \\
& {\left[\left(\boldsymbol{v}_{c} \cdot \boldsymbol{v}\right) W\right]=\left(\boldsymbol{v}_{c} \cdot \boldsymbol{v}^{-}\right) W^{-}+\left(\boldsymbol{v}_{c} \cdot \boldsymbol{v}^{+}\right) W^{+},}
\end{aligned}
$$

where $W^{-}$and $W^{+}$are one-sided finite limits of $W$ when the respective boundary $\partial M_{A}$ or $\partial M_{B}$ coinciding with $C$ is approached.

From assumed orientations of $\partial M_{A}, \partial M_{B}$, and $C$ (Figure 2) we have $\boldsymbol{v}_{A}=\boldsymbol{v}$, $\boldsymbol{v}_{B}=-\boldsymbol{v}$. In quasi-static problems discussed here the virtual translation of $C$ in $T_{x} M$ (analogous to velocity $\boldsymbol{v}_{c}$ in (23) in a real motion) is $\delta \boldsymbol{x}_{c}$, and $V=\delta \boldsymbol{x}_{c} \cdot \boldsymbol{v}$ is its component along $\boldsymbol{v}$. Then the variation of (22) with (23) leads to

$$
\delta I=\iint_{M \backslash C} \delta W \mathrm{~d} a-\int_{M \cap C} V \llbracket W \rrbracket \mathrm{d} s-\delta A,
$$

where $\llbracket W \rrbracket=W^{+}-W^{-}$is the jump of $W$ at the singular curve $C$.

The first term in (24) can be transformed with the help of (13), (12), and the Stokes theorem into

$$
\begin{aligned}
\iint_{M \backslash C} \delta W \mathrm{~d} a= & \iint_{M_{A}} \delta W \mathrm{~d} a+\iint_{M_{B}} \delta W \mathrm{~d} a \\
= & -\iint_{M \backslash C}\left\{\left.\boldsymbol{n}^{\alpha}\right|_{\alpha} \cdot \boldsymbol{v}+\left(\left.\boldsymbol{m}^{\alpha}\right|_{\alpha}+\boldsymbol{y}_{, \alpha} \times \boldsymbol{n}^{\alpha}\right) \cdot \boldsymbol{w}\right\} \mathrm{d} a \\
& +\int_{\partial M_{f}}\left(\boldsymbol{n}_{v} \cdot \boldsymbol{v}+\boldsymbol{m}_{v} \cdot \boldsymbol{w}\right) \mathrm{d} s \\
& +\int_{\partial M_{d}}\left(\boldsymbol{n}_{v} \cdot \boldsymbol{v}+\boldsymbol{m}_{v} \cdot \boldsymbol{w}\right) \mathrm{d} s \\
& -\int_{C}\left\{\llbracket \boldsymbol{n}_{v} \cdot \boldsymbol{v} \rrbracket+\llbracket \boldsymbol{m}_{v} \cdot \boldsymbol{w} \rrbracket\right\} \mathrm{d} s .
\end{aligned}
$$

Therefore, it follows from (22), (25), and (18) that the principle $\delta I=0$ is represented by

$$
\begin{aligned}
\delta I= & -\iint_{M \backslash C}\left\{\left(\left.\boldsymbol{n}^{\alpha}\right|_{\alpha}+\boldsymbol{f}\right) \cdot \boldsymbol{v}+\left(\left.\boldsymbol{m}^{\alpha}\right|_{\alpha}+\boldsymbol{y}_{, \alpha} \times \boldsymbol{n}^{\alpha}+\boldsymbol{c}\right) \cdot \boldsymbol{w}\right\} \mathrm{d} a \\
& +\int_{\partial M_{f}}\left\{\left(\boldsymbol{n}_{v}-\boldsymbol{n}^{*}\right) \cdot \boldsymbol{v}+\left(\boldsymbol{m}_{v}-\boldsymbol{m}^{*}\right) \cdot \boldsymbol{w}\right\} \mathrm{d} s \\
& +\int_{\partial M_{d}}\left(\boldsymbol{n}_{v} \cdot \boldsymbol{v}+\boldsymbol{m}_{v} \cdot \boldsymbol{w}\right) \mathrm{d} s \\
& -\int_{C}\left\{V \llbracket W \rrbracket+\llbracket \boldsymbol{n}_{v} \cdot \boldsymbol{v} \rrbracket+\llbracket \boldsymbol{m}_{v} \cdot \boldsymbol{w} \rrbracket\right\} \mathrm{d} s=0 .
\end{aligned}
$$


Vanishing of the first two rows of (26) is assured by the local equilibrium equations $(5)_{1}$. Vanishing of the line integral over $\partial M_{f}$ in (26) follows from the dynamic boundary conditions $(5)_{2}$. The line integral over $\partial M_{d}$ vanishes identically if the kinematic boundary conditions (19) are satisfied, since then $\boldsymbol{v}=\boldsymbol{w}=\mathbf{0}$ along $\partial M_{d}$.

\section{Dynamic Continuity Conditions at the Phase Interface}

Vanishing of (26) also requires the following global dynamic continuity condition to be satisfied at the curvilinear phase interface $C \subset M$ :

$$
\int_{C}\left\{V \llbracket W \rrbracket+\llbracket \boldsymbol{n}_{v} \cdot \boldsymbol{v} \rrbracket+\llbracket \boldsymbol{m}_{v} \cdot \boldsymbol{w} \rrbracket\right\} \mathrm{d} s=0 .
$$

The virtual fields $\boldsymbol{v}^{ \pm}, \boldsymbol{w}^{ \pm}$, and $V$ present in (27) are not all independent at $C$, in general. Performing the more detailed analysis of (27) we can obtain additional information about the behaviour of virtual displacements in the neighbourhood of $C$ following from assumed smoothness of the fields $\boldsymbol{y}(\boldsymbol{x})$ and $\boldsymbol{Q}(\boldsymbol{x})$.

Let us assume that $\boldsymbol{y}(\boldsymbol{x})$ and $\boldsymbol{Q}(\boldsymbol{x})$ are smooth fields in the interiors of both subregions $M_{A}$ and $M_{B}$ of $M$. Then approaching the interface $C$ the fields have one-sided finite limits

$$
\begin{array}{lllll}
\boldsymbol{y}^{-}, & \boldsymbol{F}^{-}, & \boldsymbol{Q}^{-}, & \operatorname{Grad}_{s}^{-} \boldsymbol{Q}, & \text { for } \boldsymbol{x} \rightarrow \boldsymbol{x}_{c}, \boldsymbol{x} \in M_{A}, \\
\boldsymbol{y}^{+}, & \boldsymbol{F}^{+}, & \boldsymbol{Q}^{+}, & \operatorname{Grad}_{s}^{+} \boldsymbol{Q}, & \text { for } \boldsymbol{x} \rightarrow \boldsymbol{x}_{c}, \boldsymbol{x} \in M_{B} .
\end{array}
$$

Additionally, $\boldsymbol{y}^{-}(s), \boldsymbol{Q}^{-}(s), \boldsymbol{y}^{+}(s), \boldsymbol{Q}^{+}(s)$ are continuous and continuously differentiable functions of $s$ along $C$.

The singular curve $C$ is called coherent if both $\boldsymbol{y}(\boldsymbol{x})$ and $\boldsymbol{Q}(\boldsymbol{x})$ are continuous at $C$, and incoherent if continuity of $\boldsymbol{Q}(\boldsymbol{x})$ is violated at $C$. Thus, the coherent phase interface $C$ is singular with regard to $\boldsymbol{F}$ and $\operatorname{Grad}_{s} \boldsymbol{Q}$, but not with regard to $\boldsymbol{y}$ and $\boldsymbol{Q}$ themselves. The incoherent interface $C$ is a singular curve also with regard to $\boldsymbol{Q}$.

For the coherent curvilinear phase interface $C$ we have

$$
\llbracket y \rrbracket=0, \quad \llbracket y^{\prime} \rrbracket=0, \quad \llbracket Q \rrbracket=0, \quad \llbracket Q^{\prime} \rrbracket=0 .
$$

Applying the Maxwell theorem $[44,45]$ to the fields $\delta \boldsymbol{y}$ and $\boldsymbol{F}$ at $C$ we obtain

$$
\llbracket \boldsymbol{v} \rrbracket \equiv \llbracket \delta \boldsymbol{y} \rrbracket=-V \boldsymbol{a}, \quad \llbracket \boldsymbol{F} \rrbracket=\boldsymbol{a} \otimes \boldsymbol{v},
$$

where the vector $\boldsymbol{a}$ is an amplitude of the position gradient singularity. But from $(30)_{2}$ it follows that $\boldsymbol{a}=\llbracket \boldsymbol{y}_{, \alpha} \nu^{\alpha} \rrbracket$, so that

$$
\llbracket \boldsymbol{v} \rrbracket+V \llbracket \boldsymbol{y}_{, \alpha} \nu^{\alpha} \rrbracket=\mathbf{0} \quad \text { along } C .
$$

Similarly, applying the Maxwell theorem to the fields $\delta \boldsymbol{Q}$ and $\operatorname{Grad}_{s} \boldsymbol{Q}$ at $C$ we obtain

$$
\llbracket \delta \boldsymbol{Q} \rrbracket=-V \boldsymbol{D}, \quad \llbracket \operatorname{Grad}_{s} \boldsymbol{Q} \rrbracket=\boldsymbol{D} \otimes \boldsymbol{v},
$$


where the tensor amplitude $\boldsymbol{D}$ of the rotation gradient singularity can again be found from (32) $)_{2}$ to be $\boldsymbol{D}=\llbracket \boldsymbol{Q}_{, \alpha} \nu^{\alpha} \rrbracket$, so that

$$
\llbracket \delta \boldsymbol{Q} \rrbracket+V \llbracket \boldsymbol{Q}_{, \alpha} \nu^{\alpha} \rrbracket=\mathbf{0} \quad \text { along } C .
$$

Multiplying (33) by $\boldsymbol{Q}^{\mathrm{T}}$ on the right, taking into account that $\boldsymbol{Q}^{\mathrm{T}}$ is continuous at $C$, and then using $\boldsymbol{Q}_{, \alpha} \boldsymbol{Q}^{\mathrm{T}}=\boldsymbol{\varkappa}_{\alpha} \times \mathbf{1}$, we obtain that (33) can equivalently be written as

$$
\llbracket \boldsymbol{w} \rrbracket+V \llbracket \boldsymbol{\varkappa}_{, \alpha} \nu^{\alpha} \rrbracket=\mathbf{0} \quad \text { along } C .
$$

The relations (31) and (34) are the local kinematic compatibility conditions that should be satisfied at the coherent phase interface $C$. As a result, in this case only three of five virtual fields in (27) are independent. If we represent the jumps of products in (27) by the identities

$$
\begin{aligned}
& \llbracket \boldsymbol{n}_{v} \cdot \boldsymbol{v} \rrbracket=\left\langle\boldsymbol{n}_{v}\right\rangle \cdot \llbracket \boldsymbol{v} \rrbracket+\llbracket \boldsymbol{n}_{v} \rrbracket \cdot\langle\boldsymbol{v}\rangle, \\
& \llbracket \boldsymbol{m}_{v} \cdot \boldsymbol{w} \rrbracket=\left\langle\boldsymbol{m}_{v}\right\rangle \cdot \llbracket \boldsymbol{w} \rrbracket+\llbracket \boldsymbol{m}_{v} \rrbracket \cdot\langle\boldsymbol{w}\rangle, \\
& \left\langle\boldsymbol{n}_{v}\right\rangle=\frac{1}{2}\left(\boldsymbol{n}_{v}^{-}+\boldsymbol{n}_{v}^{+}\right), \quad \text { etc. },
\end{aligned}
$$

where $\langle\cdot\rangle$ is the mean value of the limits at $C$, then the integrand of (27) with (31) and (34) can be transformed into

$$
\begin{aligned}
& V\left(\llbracket W \rrbracket-\left\langle\boldsymbol{n}_{v}\right\rangle \cdot \llbracket \boldsymbol{y}_{, \alpha} \nu^{\alpha} \rrbracket-\left\langle\boldsymbol{m}_{v}\right\rangle \cdot \llbracket \boldsymbol{\varkappa}_{, \alpha} \nu^{\alpha} \rrbracket\right) \\
& \quad+\llbracket \boldsymbol{n}_{v} \rrbracket \cdot\langle\boldsymbol{v}\rangle+\llbracket \boldsymbol{m}_{v} \rrbracket \cdot\langle\boldsymbol{w}\rangle=0 .
\end{aligned}
$$

Since $V,\langle\boldsymbol{v}\rangle$, and $\langle\boldsymbol{w}\rangle$ are now the independent virtual fields, (36) is equivalent to the following set of independent local dynamic continuity conditions at the coherent phase interface $C$ :

$$
\begin{aligned}
& \llbracket N \rrbracket \boldsymbol{v}=\mathbf{0}, \quad \llbracket \boldsymbol{M} \rrbracket \boldsymbol{v}=\mathbf{0}, \\
& \llbracket W \rrbracket-\llbracket(\boldsymbol{N} \boldsymbol{v}) \cdot \boldsymbol{y}_{, \alpha} \nu^{\alpha} \rrbracket-\llbracket(\boldsymbol{M v}) \cdot \boldsymbol{x}_{, \alpha} \nu^{\alpha} \rrbracket=0,
\end{aligned}
$$

where (4) has been used.

Let us take into account the relations

$$
\begin{array}{ll}
\boldsymbol{y}_{, \alpha} \nu^{\alpha}=\boldsymbol{F} \boldsymbol{v}, & \boldsymbol{x}_{, \alpha} \nu^{\alpha}=\left(\boldsymbol{x}_{, \alpha} \otimes \boldsymbol{a}^{\alpha}\right) \boldsymbol{v}=\boldsymbol{K} \boldsymbol{v}, \\
\boldsymbol{A}=\mathbf{1}-\boldsymbol{n} \otimes \boldsymbol{n}, & \boldsymbol{v} \cdot \boldsymbol{A} \boldsymbol{v}=1,
\end{array}
$$

where $\boldsymbol{A} \in T_{x} M \otimes T_{x} M$ is the metric tensor of $M$. Then we are able to present also $(37)_{2}$ in the coordinate-free form

$$
\boldsymbol{v} \cdot \llbracket \boldsymbol{C}_{c} \rrbracket \boldsymbol{v}=0,
$$

where

$$
\boldsymbol{C}_{c}=W \boldsymbol{A}-\boldsymbol{N}^{\mathrm{T}} \boldsymbol{F}-\boldsymbol{M}^{\mathrm{T}} \boldsymbol{K} \in T_{x} M \otimes T_{x} M
$$


is an analog in shell theory of the Eshelby tensor used in $3 \mathrm{D}$ continuum mechanics $[2,6]$.

The local dynamic continuity conditions $(37)_{1}$ and $(37)_{2}$ or (39) at the coherent curvilinear interface of the two-phase elastic shell are analogous to the thermodynamic continuity conditions at the coherent surface interface of the 3D micropolar two-phase elastic body derived in [46].

For the incoherent curvilinear phase interface $C$ the relations (29) $)_{1,2,4}$ and (31) still hold, but $(29)_{3},(33)$, and (34) are not true any more. As a result, $\boldsymbol{w}^{+}$and $\boldsymbol{w}^{-}$should now be treated as independent virtual rotation vectors. In this case the integrand of the global continuity condition (27) can be reduced to

$$
V\left(\llbracket W \rrbracket-\left\langle\boldsymbol{n}_{v}\right\rangle \cdot \llbracket \boldsymbol{y}_{, \alpha} \nu^{\alpha} \rrbracket\right)+\llbracket \boldsymbol{n}_{v} \rrbracket \cdot\langle\boldsymbol{v}\rangle+\boldsymbol{m}_{v}^{+} \cdot \boldsymbol{w}^{+}-\boldsymbol{m}_{v}^{-} \cdot \boldsymbol{w}^{-}=0,
$$

which is equivalent to the set of independent local dynamic continuity conditions along $C$

$$
\begin{aligned}
& \llbracket N \rrbracket v=\mathbf{0}, \quad \boldsymbol{M}^{ \pm} \boldsymbol{v}=\mathbf{0}, \\
& \llbracket W \rrbracket-\llbracket(N \boldsymbol{v}) \cdot \boldsymbol{F} v \rrbracket=0 .
\end{aligned}
$$

The relation $(42)_{2}$ is equivalent to

$$
v \cdot \llbracket C_{i} \rrbracket v=0,
$$

where

$$
\boldsymbol{C}_{i}=W \boldsymbol{A}-\boldsymbol{N}^{\mathrm{T}} \boldsymbol{F} .
$$

The tensor $C_{i} \in T_{x} M \otimes T_{x} M$ has no counterpart in continuum mechanics.

The first two local continuity conditions $(37)_{1,2}$ and (42) $)_{1,2}$ express just the balance of forces and couples at the curvilinear phase interface $C$. For the incoherent interface the local condition (42) $)_{2}$ indicates that the singular curve $C$ becomes here the kind of a hinge at which the resultant surface couples should vanish. The dynamic continuity conditions (39) and (43) are the additional relations which are necessary and sufficient for establishing the position of the singular curve $C$ in $M$ in the thermodynamic equilibrium state. The conditions appear here as a result of additional degrees of freedom allowed for the movable phase interface $C$ in the variational principle of stationary total potential energy.

The variational principle for the functional (22) of total potential energy as well as the dynamic continuity conditions (27), (37), and (42) for elastic shells undergoing phase transitions seem to be new in the literature.

Summarising, the equlibrium boundary value problem of the general nonlinear theory of elastic shells with phase transitions consists of the local equilibrium equations $(5)_{1}$, the dynamic boundary conditions $(5)_{2}$, the kinematic boundary conditions (19), the kinematic relations (11), and the constitutive equations (15) supplemented by the local continuity conditions (37) and (39) at the coherent interface or (42) 1 and (43) at the incoherent interface, respectively. 


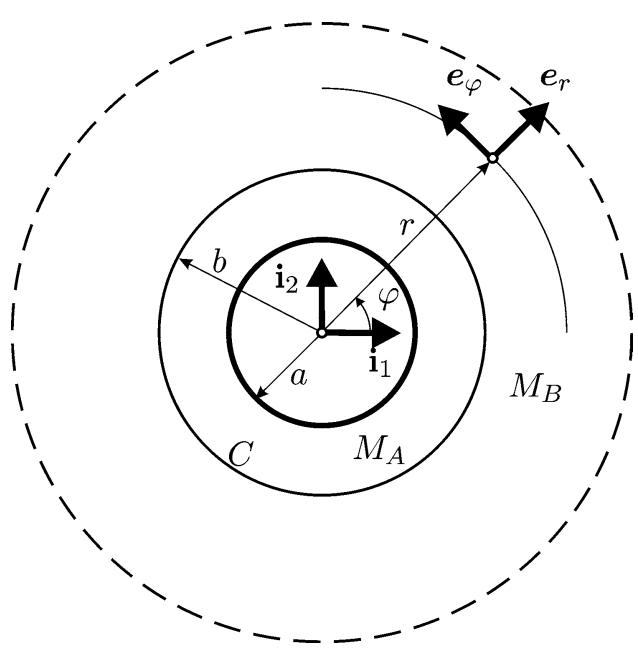

Figure 3. Two-phase plate with a hole.

\section{Example}

Let us solve a simple axisymmetric plane problem of phase transition in an infinite plate with a central hole of radius $a$, Figure 3 . It is assumed that in the undeformed state the whole plate consists of one material phase marked by " $B$ ". Then to the hole boundary the radial translation $\boldsymbol{u}^{*}=u^{*} \boldsymbol{e}_{r}$ with a constant magnitude $u^{*}$ is prescribed. As a result, in the deformed equilibrium state of phase transition there may appear a new material phase marked by " $A$ " in the region $a<r<b$. Our goal is to find the radius $b$ of the interface circle $C$.

In the polar coordinate system $\theta^{1}=r, \theta^{2}=\varphi$ the axisymmetric plane deformation can be described by

$$
\begin{aligned}
& \boldsymbol{y}=y(r) \boldsymbol{e}_{r}, \quad \boldsymbol{u}=u(r) \boldsymbol{e}_{r}=(y(r)-1) \boldsymbol{e}_{r}, \quad \boldsymbol{Q}=\mathbf{1}, \\
& \boldsymbol{e}_{r}=\cos \varphi \mathbf{i}_{1}+\sin \varphi \mathbf{i}_{2}, \quad \boldsymbol{e}_{\varphi}=-\sin \varphi \mathbf{i}_{1}+\cos \varphi \mathbf{i}_{2},
\end{aligned}
$$

where $\boldsymbol{e}_{r}$ and $\boldsymbol{e}_{\varphi}$ are the unit base vectors, and $y(r)$ or $u(r)$ are unknown, Figure 3.

The strain and bending measures corresponding to (45) are

$$
\begin{array}{lrl}
\boldsymbol{\varepsilon}_{r}=\frac{\mathrm{d} u}{\mathrm{~d} r} \boldsymbol{e}_{r} \equiv u^{\prime} \boldsymbol{e}_{r}, & \boldsymbol{\varepsilon}_{\varphi}=u \boldsymbol{e}_{\varphi}, & \boldsymbol{\varkappa}_{r}=\boldsymbol{\varkappa}_{\varphi} \equiv \mathbf{0}, \\
E_{r r}=u^{\prime}, & E_{\varphi \varphi}=u, & E_{r \varphi}=E_{\varphi r}=0, \\
K_{r r}=K_{\varphi \varphi}=K_{r \varphi}=K_{\varphi r}=0, &
\end{array}
$$

so that the quadratic strain energy density (16) takes the form

$$
\begin{aligned}
W & =\left(C_{1}+C_{2}\right)\left(E_{r r}^{2}+\frac{E_{\varphi \varphi}^{2}}{r^{2}}\right)+C_{3}\left(E_{r r}+\frac{E_{\varphi \varphi}}{r}\right)^{2} \\
& =\left(C_{1}+C_{2}\right)\left(u^{\prime 2}+\frac{u^{2}}{r^{2}}\right)+C_{3}\left(u^{\prime}+\frac{u}{r}\right)^{2} .
\end{aligned}
$$


Within the axisymmetric deformation state described by (45) other material constants $C_{4}$ and $D_{n}$ present in (16) are not required.

Differentiating (47) we obtain the constitutive equations $\boldsymbol{M} \equiv \mathbf{0}$ and

$$
\begin{aligned}
N & =N_{r} \boldsymbol{e}_{r} \otimes \boldsymbol{e}_{r}+N_{\varphi} \boldsymbol{e}_{\varphi} \otimes \boldsymbol{e}_{\varphi}, \\
N_{r} & =\frac{\partial W}{\partial E_{r r}}=2\left(C_{1}+C_{2}\right) u^{\prime}+2 C_{3}\left(u^{\prime}+\frac{u}{r}\right), \\
N_{\varphi} & =\frac{\partial W}{\partial E_{\varphi \varphi}}=2\left(C_{1}+C_{2}\right) \frac{u}{r}+2 C_{3}\left(u^{\prime}+\frac{u}{r}\right) .
\end{aligned}
$$

With no external resultant surface forces and couples, the equilibrium equations (6) reduce here to only one ordinary differential equation

$$
N_{r}^{\prime}+\frac{1}{r}\left(N_{r}-N_{\varphi}\right)=0
$$

which with the help of (49) can be transformed into

$$
\left(C_{1}+C_{2}\right) u^{\prime \prime}+C_{3}\left(u^{\prime \prime}+\frac{u^{\prime}}{r}-\frac{u}{r^{2}}\right)+\frac{1}{r}\left(C_{1}+C_{2}\right)\left(u^{\prime}-\frac{u}{r}\right)=0,
$$

and after further transformation into

$$
\left(C_{1}+C_{2}+C_{3}\right)\left(u^{\prime}+\frac{u}{r}\right)^{\prime}=0 .
$$

The solution of (50) takes the form

$$
u=u_{0} r+\frac{u_{1}}{r},
$$

where $u_{0}$ and $u_{1}$ are integration constants.

Let us assume that the strain energy densities in the two material phases differ only by values of the elastic constants in (47)

$$
W_{A, B}=\left(C_{1}^{A, B}+C_{2}^{A, B}\right)\left(u^{\prime 2}+\frac{u^{2}}{r^{2}}\right)+C_{3}^{A, B}\left(u^{\prime}+\frac{u}{r}\right)^{2} .
$$

Kinematic boundary conditions are given by

$$
u_{A}(a)=u^{*} \quad \text { and } \quad u_{B}(r) \rightarrow 0 \quad \text { for } r \rightarrow \infty .
$$

Continuity conditions of the type $(37)_{2}$ and $(42)_{2}$ for the resultant couples are identically satisfied at $C$, while the continuity conditions for translations (29) $)_{1}$ and stress resultants $(37)_{1}$ are given by

$$
u^{-}(b)=u^{+}(b), \quad N_{r}^{-}(b)=N_{r}^{+}(b) .
$$


Introducing (51) into (52) and (53) we obtain the following algebraic system of equations for the integration constants $u_{0}^{ \pm}$and $u_{1}^{ \pm}$:

$$
\begin{aligned}
& u_{0}^{-}+\frac{u_{1}^{-}}{a^{2}}=\frac{u^{*}}{a}, \quad u_{0}^{-}+\frac{u_{1}^{-}}{b^{2}}=u_{0}^{+}+\frac{u_{1}^{+}}{a^{2}} \\
& \left(C_{1}^{A}+C_{2}^{A}\right)\left(u_{0}^{-}-\frac{u_{1}^{-}}{b^{2}}\right)+2 C_{3}^{A} u_{0}^{-}=\left(C_{1}^{B}+C_{2}^{B}\right)\left(u_{0}^{+}-\frac{u_{1}^{+}}{b^{2}}\right)+2 C_{3}^{B} u_{0}^{+}, \\
& \quad u_{1}^{+}=0 .
\end{aligned}
$$

Solution of (54) allows one to express these constants through $u^{*}$ and $b$.

The radius $b$ can now be found from the continuity conditions (39) or (43) which in this case coincide. Condition (39) can be reduced to

$$
W^{+}-\left(u^{\prime+}+1\right) N_{r}=W^{-}-\left(u^{\prime-}+1\right) N_{r}
$$

or, after introducing the solution (51), to

$$
\begin{aligned}
& \left(C_{1}^{B}+C_{2}^{B}\right)\left[\left(u_{0}^{+}-\frac{u_{1}^{+}}{b^{2}}\right)^{2}+\left(u_{0}^{+}+\frac{u_{1}^{+}}{b^{2}}\right)^{2}\right]+4 C_{3}^{B}\left(u_{0}^{+}\right)^{2} \\
& -\left[2\left(C_{1}^{B}+C_{2}^{B}\right)\left(u_{0}^{+}-\frac{u_{1}^{+}}{b^{2}}\right)+4 C_{3}^{B} u_{0}^{+}\right]\left(u_{0}^{+}-\frac{u_{1}^{+}}{b^{2}}+1\right) \\
& =\left(C_{1}^{A}+C_{2}^{A}\right)\left[\left(u_{0}^{-}-\frac{u_{1}^{-}}{b^{2}}\right)^{2}+\left(u_{0}^{-}-\frac{u_{1}^{-}}{b^{2}}\right)^{2}\right]+4 C_{3}^{A}\left(u_{0}^{-}\right)^{2} \\
& \quad-\left[2\left(C_{1}^{A}+C_{2}^{A}\right)\left(u_{0}^{-}-\frac{u_{1}^{-}}{b^{2}}\right)+4 C_{3}^{A} u_{0}^{-}\right]\left(u_{0}^{-}-\frac{u_{1}^{-}}{b^{2}}+1\right) .
\end{aligned}
$$

Solving (54) and (55) we obtain $b$ as a function of $u^{*}$.

Let us discuss a particular case of the plate material for which $C_{2}^{A, B}=C_{3}^{A, B}=0$. Then equation (55) for $b$ can be considerably simplified to

$$
\frac{u^{*} x(k-1)(k+1)\left(\left(u^{*}+a\right) / a x k-k-x-1\right)}{a(x k-x-k-1)^{2}}=0,
$$

where $x=a^{2} / b^{2}, k=C_{1}^{B} / C_{1}^{A}$.

Equation (56) is identically satisfied by $k=1$, that is in the case of equal elastic constants $C_{1}^{A}$ and $C_{1}^{B}$. In such a case there is no phase transition in the plate at all.

If $k \neq 1$, then $b$ is given by the relation

$$
b=a \sqrt{\frac{\left(u^{*} / a+1\right) k-1}{k+1}} .
$$

The real solution of (57) exists only if $u^{*}>u_{0}^{*} \equiv 2 a / k$. The solution indicates that within $0<u^{*}<u_{0}^{*}$ there is in the plate only one material phase " $B$ ". When the assumed boundary translation reaches the critical value $u^{*}=u_{0}^{*}$ the second 


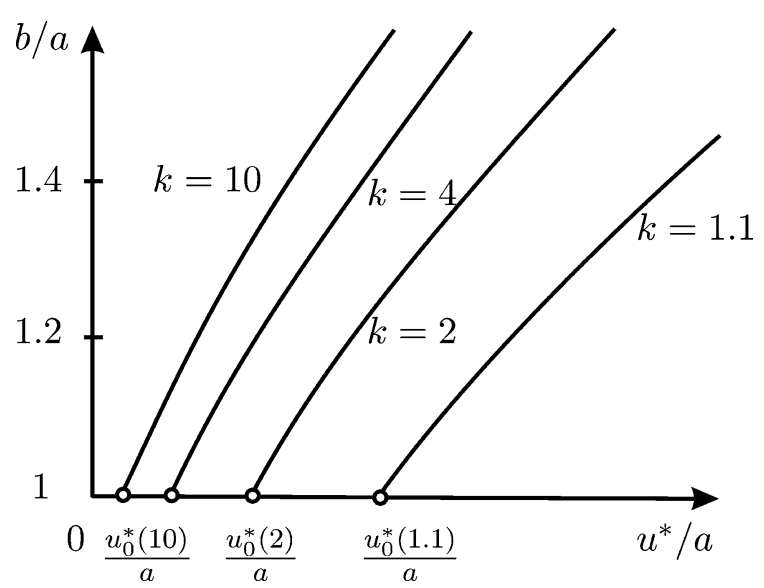

Figure 4. Phase diagram for two-phase plate.

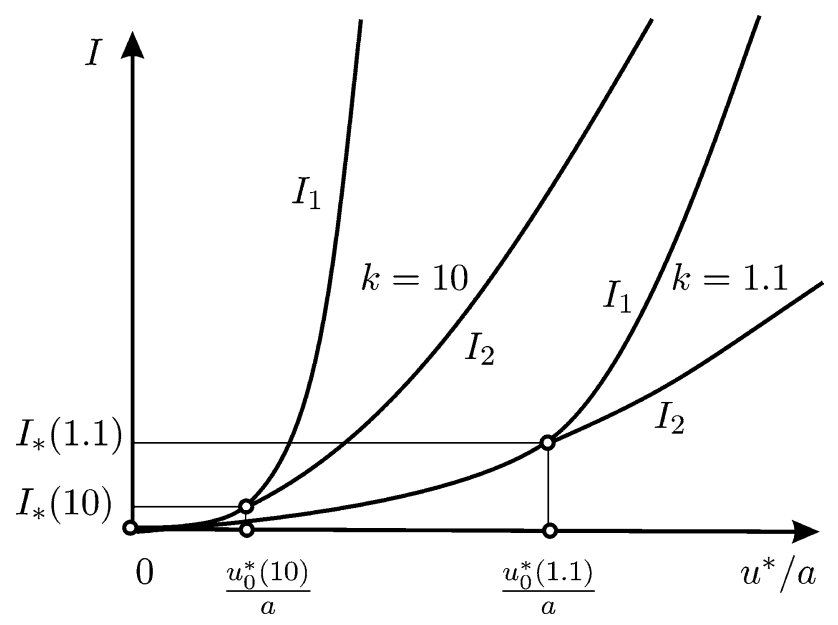

Figure 5. Total energies for two-phase solution $I_{2}$ and for one-phase solution $I_{1}$.

more "soft" material phase " $A$ " is created with smaller modulus of elasticity than that of the phase " $B$ ". Finally, when $u^{*}>u_{0}^{*}$ the plate consists of two regions with two different material phases separated by the circular interface $C$ of radius $b$. The value of $b$ depends upon the ratio $k$ between the material constants $C_{1}^{B}$ and $C_{2}^{A}$, Figure 4 , where $u_{0}^{*}(k) / a$ are dimensionless values corresponding to the respective $k$.

It is easy to see that the solution corresponding to the two-phase equilibrium state is energetically consistent. Let us calculate the value of the functional $I$ on the two-phase solution (51) and (57) as well as on the corresponding one-phase solution for two values of $k=10$, and $k=1.1$. Then the total potential energy $I_{2}$ corresponding to the two-phase equilibrium state is always lower than $I_{1}$ corresponding to the one-phase state, see Figure 5. 


\section{Conclusions}

In this paper a boundary value problem has been proposed for the mechanical description of the phase transformation phenomena in elastic shells. The nonlinear shell model has been based on the exact trough-the-thickness integration of 3D global equilibrium conditions for the total force and total torque, with an energetically exact shell kinematics established by the virtual work identity. The phase transition has been assumed to occur at the singular surface curve; its position is not known in advance. The equilibrium problem of elastic shells with phase transitions has been stated in the weak form as the stationary problem for the functional of total potential energy. Stationary conditions of the functional give us known local equilibrium conditions everywhere outside the interface, together with additional new local dynamic continuity conditions to be satisfied at the interface. The continuity conditions result from additional degrees of freedom associated with the unknown position of the interface.

The shell model used here is the most general one available. Therefore, all possible simplified versions of shell theory with phase transitions can now be derived as special cases of our results. One has only to introduce appropriate geometric and/or physical constraints and to omit some terms assumed to be small. Among such simplified versions let us mention, for example, the fully nonlinear, only the geometrically nonlinear, or the entirely linearised theories based on the Timoshenko-Reissner type, the Kirchhoff-Love type, or the membrane type shell model.

The functional (22) of total potential energy does not take into account some physical effects of phase transition in shells associated with properties of the curvilinear interface itself. Besides, by (22) we can model only quasi-static shell problems with phase transitions not depending upon temperature. The phase transitions in shells based on various appropriate generalisations of (22) should be discussed separately.

\section{Acknowledgements}

The research was partially supported by The Józef Mianowski Fund and The Competition Center of Natural Sciences in Saint-Petersburg State University (grant No. E02-4.0-91) as well as by the Polish State Committee for Scientific Research (grant KBN No. 5 T07A 008 25).

\section{References}

1. J.W. Gibbs, On the equilibrium of heterogeneous substances. Trans. Connecticut Acad. Sci. 3 (1875/1878) 108-248, (1875) 343-524. Reprinted in: The Collected Works of J. Willard Gibs. Longmans/Green, New York (1928) pp. 55-353.

2. M. Grinfeld, Thermodynamics Methods in the Theory of Heterogeneous Systems. Longman, Harlow (1991). 
3. A. Romano, Thermodynamics of Phase Transitions in Classical Field Theory. World Scientific, Singapore (1993).

4. M.E. Gurtin, Thermomechanics of Evolving Phase Boundaries in the Plane. Clarendon Press, Oxford (1993).

5. G.A. Maugin, Material Inhomogeneities in Elasticity. Chapman and Hall, London (1993).

6. M.E. Gurtin, Configurational Forces as Basic Concepts of Continuum Physics. Springer, Berlin (2000).

7. K. Bhattacharya and R.D. James, A theory of thin films of martensitic materials with applications to microactuators. J. Mech. Phys. Solids 36 (1999) 531-576.

8. R.D. James and R. Rizzoni, Pressurized shape memory thin films. J. Elasticity 59 (2000) $399-436$.

9. J.G. Simmonds, The nonlinear thermodynamical theory of shells: descent from 3-dimensions without thickness expansion. In: E.L. Axelrad and F.A. Emmerling (eds), Flexible Shells, Theory and Applications. Springer, Berlin (1984) pp. 1-11.

10. A. Libai and J.G. Simmonds, The Nonlinear Theory of Elastic Shells, 2nd ed. Cambridge Univ. Press, Cambridge (1998).

11. S.S. Antman, Nonlinear Problems of Elasticity. Springer, New York (1995).

12. A. Libai and J.G. Simmonds, Nonlinear elastic shell theory. Adv. Appl. Mech. 23 (1983) 271371.

13. J. Makowski and H. Stumpf, Buckling equations for elastic shells with rotational degrees of freedom undergoing finite strain deformation. Internat. J. Solids Struct. 26 (1990) 353-368.

14. W. Pietraszkiewicz, Teorie nieliniowe powłok. In: Cz. Woźniak (ed.), Mechanika Sprężystych Ptyt i Powtok. Wyd. Nauk. PWN, Warszawa (2001) pp. 424-497.

15. J. Chróścielewski, J. Makowski and W. Pietraszkiewicz, Statyka i Dynamika Powłok Wieloptatowych: Nieliniowa Teoria i Metoda Elementów Skończonych. Wydawnictwo IPPT PAN, Warszawa (2004).

16. E. Cosserat and F. Cosserat, Théorie des Corps Deformables. Herman et Flis, Paris (1909); English translation: NASA TT F-11, 561. NASA, Washington, DC (1968).

17. H. Altenbach and P.A. Zhilin, The theory of elastic thin shells (in Russian). Adv. in Mech. 11 (1988) 107-148.

18. L.M. Zubov, Nonlinear Theory of Dislocations and Disclinations in Elastic Bodies. Springer, New York (1997).

19. L.M. Zubov, Semi-inverse solutions in nonlinear theory of elastic shells. Arch. Mech. 53 (2001) 599-610.

20. L.M. Zubov, General solutions to the nonlinear static problem for elastic shells. Doklady Phys. 47 (2002) 74-77.

21. V.A. Eremeyev and L.M. Zubov, General nonlinear theory of elastic micropolar shells (in Russian). Izv. Vuzov Severo-Kavk. Region, Ser. Est. Nauki, Spetsvypusk (2003) 124-169.

22. J. Chróścielewski, Rodzina elementów skończonych klasy $C^{0}$ w nieliniowej sześcioparametrowej teorii powłok. Zesz. Nauk. Politechniki Gdańskiej Nr. 540, Bud. Ląd. LIII (1996) $1-291$.

23. J. Chróścielewski, J. Makowski and H. Stumpf, Finite element analysis of smooth, folded and multi-shell structures. Comput. Methods Appl. Mech. Engrg. 141 (1997) 1-46.

24. J. Chróścielewski, J. Makowski and W. Pietraszkiewicz, Nonlinear dynamics of flexible shell structures. Comput. Assisted Mech. Engrg. Sci. 9 (2002) 341-357.

25. I. Lubowiecka and J. Chróścielewski, On dynamics of flexible branched shell structures undergoing large overall motion using finite elements. Computers Structures 80 (2002) 891-898.

26. C. Truesdell and W. Noll, The nonlinear field theories of mechanics. In: S. Flügge (ed.), Handbuch der Physik, Vol. III/3. Springer, Berlin (1965) pp. 1-602. 
27. J. Makowski and W. Pietraszkiewicz, Thermomechanics of Shells with Singular Curves. Zeszyty Naukowe IMP PAN Nr 528(1487), Gdańsk (2002) pp. 1-100.

28. M.E. Gurtin and A.I. Murdoch, A continuum theory of elastic material surfaces. Arch. Rational Mech. Anal. 57 (1975) 291-323.

29. A.I. Murdoch, A coordinate free approach to surface kinematics. Glasgow Math. J. 32 (1990) 299-307.

30. J. Makowski, W. Pietraszkiewicz and H. Stumpf, Jump conditions in the nonlinear theory of thin irregular shells. J. Elasticity 54 (1999) 1-26.

31. J.L. Ericksen and C. Truesdell, Exact theory of stress and strain in rods and shells. Arch. Rational Mech. Anal. 1 (1957) 295-323.

32. P.M. Naghdi, The theory of plates and shells. In: S. Flügge (ed.), Handbuch der Physik, Vol. VIa/2. Springer, Berlin (1972) pp. 425-640.

33. M.B. Rubin, Cosserat Theories: Shells, Rods and Points. Kluwer Academic Publishers, Dordrecht (2000).

34. Ch.M. Mushtari and K.Z. Galimov, Nonlinear Theory of Thin Elastic Shells (in Russian). Tatknigoizdat, Kazan' (1957); English translation: The Israel Program for Sci. Transl. (1961).

35. W.T. Koiter, On the nonlinear theory of thin elastic shells. Proc. Koninkl. Ned. Acad. Wetensch. B 69 (1966) 1-54.

36. W. Pietraszkiewicz, Geometrically nonlinear theories of thin elastic shells. Adv. in Mech. 12 (1989) 51-130.

37. W. Pietraszkiewicz, On using rotations as primary variables in the nonlinear theory of thin irregular shells. In: D. Durban et al. (eds), Advances in the Mechanics of Plates and Shells. Kluwer Academic Publishers, Dordrecht (2001) pp. 245-258.

38. W. Pietraszkiewicz, Finite Rotations and Lagrangean Description in the Non-Linear Theory of Shells. Polish Sci. Publ., Warszawa/Poznań (1979).

39. M. Kleiber and Cz. Woźniak, Nonlinear Mechanics of Structures. Kluwer Academic Publishers, Dordrecht (1991).

40. A.J.M. Spencer, Theory of invariants. In: A.C. Eringen (ed.), Continuum Physics, Vol. 1. Academic Press, New York (1971) pp. 239-353.

41. J. Badur, Nieliniowa analiza powłok sprężystych według teorii drugiego przybliżenia do energii odkształcenia. Rozprawa doktorska, Instytut Maszyn Przepływowych PAN, Gdańsk (1984).

42. M.E. Gurtin and M.E. Jabbour, Interface evolution in three dimensions with curvaturedependent energy and surface diffusion: Interface-controlled evolution, phase transitions, epitaxial growth of elastic films. Arch. Rational Mech. Anal. 163 (2002) 171-208.

43. R. Abeyaratne and J.K. Knowles, A note on the driving traction acting on a propagating interface: Adiabatic and non-adiabatic processes of a continuum. Trans. ASME J. Appl. Mech. 67 (2000) 829-831.

44. C. Truesdell, Rational Thermodynamics. Springer, New York (1984).

45. C. Truesdell, A First Course in Rational Continuum Mechanics. Academic Press, New York (1977).

46. V.A. Eremeyev and L.M. Zubov, The conditions of phase equilibrium in nonlinear media with microstructure (in Russian). Proc. Russian Acad. Sci. 326 (1992) 968-971. 